\title{
Comparison of direct versus concentrated smear microscopy in detection of pulmonary tuberculosis
}

\author{
Mohammad Khaja Mafij Uddin', Md Raihan Chowdhury', Shahriar Ahmed', Md Toufiq Rahman',
} Razia Khatun ${ }^{1}$, Frank van Leth² and Sayera Banu ${ }^{1 *}$

\begin{abstract}
Background: Sputum smear microscopy is fast and inexpensive technique for detecting tuberculosis (TB) in high incidence areas but has low sensitivity. Physical and chemical sputum processing along with centrifugation have been found to show promise in overcoming this limitation. Our objective was to compare the sensitivity of smear microscopy obtained with smears made directly from respiratory specimens to those from concentrated specimens.

Methods: By active screening, 915 TB suspects were identified from Dhaka Central Jail and sputum specimens were aseptically collected. Direct smears were prepared by taking a small portion of the purulent part of the sputum with a sterile loop. The specimens were then processed by a standard $\mathrm{N}$-acetyl-L-cysteine- $\mathrm{NaOH}$ digestion-decontamination method to prepare concentrated specimens. Both smears were then air dried, heat fixed, and stained by the Ziehl-Neelsen staining technique. The stained slides were examined under oil immersion and were graded following International Union Against Tuberculosis and Lung Diseases guidelines. All the specimens were inoculated into Lowenstein-Jensen (L-J) media and culture results were considered as gold standard to calculate sensitivity.

Results: Of 915 specimens, 73 (8\%) specimens were positive both on direct and concentrated methods, one sample was positive on direct microscopy but was negative on concentrated method. An extra 14 (1.5\%) samples were positive on concentrated method which were negative on direct smear. In L-J media 105 specimens were found positive for TB bacilli and of them, 74 (70.5\%) and 87 (82.9\%) were positive in direct and concentrated smear, respectively. The sensitivity of direct and concentrated smear microscopy was different when using positive culture as the gold standard (71\% vs. 83\%).

Conclusions: The results showed that concentrated technique increases the sensitivity of microscopy up to $12 \%$. Therefore, the national programs in high TB burden countries may consider incorporating the technique into their guidelines at least in the district and higher level laboratories to improve case finding strategy.
\end{abstract}

Keywords: Tuberculosis, Sensitivity, Direct vs. Concentrated smear microscopy, Sputum smear microscopy

\section{Background}

Tuberculosis (TB) continues to be a major public health threat worldwide despite the availability of many highly sensitive diagnostic tools and highly efficacious treatment for decades. It is more of a threat in the developing world. There were an estimated 8.8 million new cases (incidence) of TB (range, 8.5 million-9.2 million) globally in 2010, 1.1 million deaths (range, 0.9 million-1.2

\footnotetext{
* Correspondence: sbanu@icddrb.org

'International Centre for Diarrhoeal Disease Research, Bangladesh

Full list of author information is available at the end of the article
}

million) among human immunodeficiency virus (HIV)negative cases of $\mathrm{TB}$ and an additional 0.35 million deaths (range, 0.32 million-0.39 million) among people who were HIV-positive. This makes TB the second leading cause of death among the infectious diseases [1]. A staggering $95 \%$ of these cases and deaths occur in the developing countries $[2,3]$.

Early diagnosis of TB is crucial both clinically and epidemiologically. It is essential to ensure proper and early identification of cases, and good treatment outcomes to be able to limit its transmission and obtain successful
C Biomed Central

(C) 2013 Uddin et al.; licensee BioMed Central Ltd. This is an Open Access article distributed under the terms of the Creative Commons Attribution License (http://creativecommons.org/licenses/by/2.0), which permits unrestricted use, distribution, and reproduction in any medium, provided the original work is properly cited. 
TB control [4]. The gold standard for pulmonary TB diagnosis is culture of sputum in liquid media. However, due to lack of access to culture facilities and the long turn-around times involved with sputum culture, most programmes use direct Ziehl-Neelsen (Z-N) microscopy for detection of acid-fast bacilli (AFB) in sputum smears as their main diagnostic tool. In this method, the sputum specimens are smeared directly on to the slides without any processing and subjected to $\mathrm{ZN}$ staining. AFB microscopy is believed to be the most practical and fastest technique in establishing a diagnosis of pulmonary $\mathrm{TB}$, especially in developing countries where most of the TB cases live $[4,5]$. Studies have shown that direct smear microscopy is highly specific in settings where TB is more prevalent $[6,7]$. Though AFB microscopy is simple, inexpensive and provides rapid result, it has some limitations. The threshold for detection of AFB in sputum samples under optimal conditions is between $10^{4}$ and $10^{5}$ bacilli per ml. The sensitivity and specificity of AFB microscopy is low when compared to culture method. In some studies it has been shown that this technique has a low sensitivity, $22-43 \%$ for a single smear [8] and up to $60 \%$ under optimal conditions $[9,10]$ when compared with that of cultures. Sensitivity is even more reduced if samples are of poor quality, which is often the case in children and HIV-coinfected patients $[11,12]$. Although all mycobacterial species are acid fast, this assay is highly specific for $M$. tuberculosis in countries where TB is endemic [13].

Microscopy clearly has many advantages when it comes to speed and feasibility, and if sensitivity could be improved it has the potential to become an even more valuable tool for National TB Control Programmes (NTPs) around the world. In the last decade many researchers have suggested that the performance of sputum smear microscopy can be significantly improved if sputum is liquefied with chemical reagents and then concentrated by centrifugation or sedimentation prior to acid-fast staining $[14,15]$. Out of them, the technique using $\mathrm{N}$-acetyl-L-cysteine (NALC) with $2 \%$ sodium hydroxide $(\mathrm{NaOH})$ is considered to be the best. NALC acts as a strong mucus digester and the smear processed by it has less debris and a greater concentration of AFB [16]. This method has been found to increase the sensitivity of microscopy substantially [10]. However, it requires some level of staff training, increases time needed for diagnosis, and requires some level of biosafety arrangement to ensure the security of the lab personnel. Due to resource constraints, it is not applied in the majority of TB laboratories in developing countries $[17,18]$.

Another chemical, sodium hypochlorite, usually known as household bleach is considered as an ideal chemical processing agent for use in low income countries. It is widely available and inexpensive, and its disinfectant properties could improve infection control in laboratories lacking biosafety facilities. It is reported that bleach increase the sensitivity of smear microscopy through the digestion of mucus and debris in sputum and resulting in clearer microscopy field [19]. One of the notable disadvantages of bleach sedimentation is that a bleach treated sample cannot be used for mycobacterial culture, as the bleach kills $M$. tuberculosis.

Another recent development in smear microscopy is the advent of fluorescence microscopy (FM). Examination of sputum smears stained with $\mathrm{Z}-\mathrm{N}$ requires on an average 5-10 minutes, consuming considerable working hours from the laboratories with limited resources. The newer alternative technique to $\mathrm{Z}-\mathrm{N}$ smear microscopy, FM is known to increase the sensitivity (10\% higher) when compared with Z-N microscopy methods while speeding up the whole process to consume much lesser time [20]. Fluorescent AFB can be seen at lower magnification than $\mathrm{Z}-\mathrm{N}$ stained AFB. FM smears can be examined in a fraction (about 25\%) of the time needed for Z-N smears as well. Recent development of simple FM systems based on light-emitting diodes (LED-FM) which have long lifespans, do not produce UV light, and have minimal power requirements could facilitate the implementation of FM in high burden and limited resources countries [21].

The use of sputum smear as a screening procedure for the diagnosis of pulmonary $\mathrm{TB}$ has recently been criticized following the finding by several large laboratories that up to $55 \%$ of specimens with positive smear failed to grow in culture while $30 \%$ are smear negative but culture positive $[22,23]$. The use of different smearing techniques varies from one laboratory to another. In most of the low and middle income countries the direct smear technique is used and only a few uses the concentrated method [23]. Though the resource needed to practice concentrated smearing technique remains a problem in many laboratories, it has been shown that concentration and liquefaction improve the sensitivity of the AFB smear microscopy and can contribute significantly to achieve better accuracy of diagnosis [24].

As microscopy is the mainstay of TB diagnosis in our country, in this study we wanted to compare the sensitivity obtained with smears for detection of AFB prepared directly from respiratory specimens (direct AFB smears) to that obtained with the parallel smears prepared from concentrate of the specimens (concentrated AFB smears) in our country context. This information may be of great help to the NTP in formulating effective TB control guidelines.

\section{Methods}

\section{Specimen processing and culture}

A total of 915 sputum specimens were aseptically collected from prisoners of the Dhaka Central Jail who were 
suspected to have pulmonary TB disease on the basis of their presenting symptoms. A suspect was defined as an individual if he/she had persistent cough for more than three weeks, and/or evening rise of temperature for more than two weeks, and/or body mass index (BMI) less than 16. A brief study questionnaire was used to identify the suspects among the inmates in Dhaka Central Jail, the largest prison in Bangladesh. The collected specimens were transported in specimen transportation box (cool box) to the Tuberculosis Laboratory at International Centre for Diarrhoeal Disease Research, Bangladesh (icddr,b) for AFB microscopy and culture.

Specimens were scored as saliva or sputum on the basis of visual examination. Suspects were requested to give another specimen in case of saliva specimens. This approach has been assessed as being feasible for the detection of specimens with a high probability of being positive a culture in a clinical setting. The use if this approach in a population-based screening programme outside the clinical setting is not to be recommended [25]. Direct smears were prepared by taking a small portion of the purulent part of the sputum with a sterile loop. The specimens were then processed by a standard NALC-NaOH digestion-decontamination method; briefly, an equal volume of $2 \%$ $\mathrm{NaOH}$ and $1.45 \%$ sodium citrate containing $0.5 \%$ NALC were added to each tube. The contents within the tubes were then mixed by vortexing and then incubated at room temperature for 15 minutes. The tubes were then shaken by hand at regular intervals. Phosphate-buffered saline ( $\mathrm{pH}$ 6.8) were added up to $45 \mathrm{ml}$ and then centrifuged at 3000X g for 15 minutes. The supernatant were carefully poured off, the resulting sediments were then resuspended in $1.5 \mathrm{ml}$ of phosphate-buffered saline, and the suspensions were used to prepare smear (concentrated smear) and to inoculate into Lowenstein-Jensen (L-J) culture media. Though the World Health Organization (WHO) recommended gold standard in TB diagnosis is culture of sputum in liquid media, in our study we have used culture in solid media (L-J) as gold standard as no liquid culture facility was available in our country during the study period.

\section{AFB smear and microscopy}

Smears made from original specimens and/or from the concentrated specimens were air dried, heat fixed, and stained by the ZN staining technique. The stained slides were examined under oil immersion $(1,000 x$ lens objective), and they were reported negative when no AFB were seen in at least 100 microscopic fields. Smears were graded positive [17] for any of the following observations: when 1 to 9 AFB were seen in 100 microscopic fields (scored as scanty positive), when 10 to 99 AFB were seen in 100 fields (scored as $1+$ ), when 1 to $10 \mathrm{AFB}$ were seen per field in at least 50 fields (scored as 2+), and when more than 10 AFB were seen per field in at least 20 fields (scored as 3+). Direct smear and concentrated smears were examined separately by two laboratory technicians without knowing the results of each other. A total of $10 \%$ slides from both methods were reexamined by the laboratory supervisor and his classification was final in case of discrepancies.

All the specimens inoculated into L-J media were incubated at $37^{\circ} \mathrm{C}$ for 6 to 8 weeks in a vertical position for the better development of individual colonies. When small and buff colored colonies grew on LJ medium, the sample was considered as positive. Contaminated cultures (e.g. growth of moulds, and also those in which the medium had liquefied or turned dark green) were discarded.

The study protocol was approved by the Ethical Review Committee of International Centre for Dirrhoeal Disease Research, Bangladesh (icddr,b). All study subjects were enrolled in the study only after they had provided informed written consent.

\section{Data entry and analysis}

All calculations were performed using SPSS, version 17.0 for Windows. Sensitivity and specificity were calculated for each diagnostic technique and expressed as percentages at $95 \%$ confidence intervals. The McNemar test was used to compare the sensitivity of the different tests.

\section{Results}

Out of 915 specimens 74 (8.1\%) were found to be AFB positive and 841 (91.9\%) were found AFB negative by direct AFB smear. Among the positive specimens, 9 (1\%) were scanty positive, $19(2 \%)$ were $1+, 27(3 \%)$ were $2+$ and $19(2 \%)$ were $3+$. In contrast, 87 (9.5\%) were found to be AFB positive and 828 (90.5\%) were AFB negative when smear prepared from the concentrated specimens. Among the positive specimens $13(2 \%), 18(2 \%), 26(3 \%), 30(3 \%)$ were scanty positive, $1+, 2+$ and $3+$ respectively (Table 1 ).

Grading of AFB between the slides prepared from the same sample using direct and concentrated method was

\begin{tabular}{|c|c|c|c|c|c|c|}
\hline \multirow{2}{*}{$\begin{array}{l}\text { Direct } \\
\text { smear } \\
\text { results }\end{array}$} & \multicolumn{5}{|c|}{$\begin{array}{l}\text { No. of specimens with the following } \\
\text { concentrated smear results: }\end{array}$} & \multirow[t]{2}{*}{ Total } \\
\hline & $\begin{array}{l}\text { Negative } \\
\mathrm{n}(\%)\end{array}$ & $\begin{array}{l}\text { Scanty } \\
\text { n (\%) }\end{array}$ & $\begin{array}{l}1+ \\
n(\%)\end{array}$ & $\begin{array}{l}2+ \\
\mathrm{n}(\%)\end{array}$ & $\begin{array}{l}3+ \\
\mathrm{n}(\%)\end{array}$ & \\
\hline Negative & 827 (98.3) & $11(1.3)$ & $3(0.4)$ & & & 841 \\
\hline Scanty & $1(11.1)$ & $2(22.2)$ & $6(66.7)$ & & & 9 \\
\hline $1+$ & & & $8(42.1)$ & $10(52.6)$ & $1(5.3)$ & 19 \\
\hline $2+$ & & & $1(3.7)$ & $16(59.3)$ & $10(37.0)$ & 27 \\
\hline $3+$ & & & & & 19 (100) & 19 \\
\hline Total & 828 & 13 & 18 & 26 & 30 & 915 \\
\hline
\end{tabular}


compared. Of the 915 specimens, 73 (8\%) specimens were positive both on direct and concentrated methods, one sample was positive on direct microscopy but was negative on concentrated method. An extra 14 (1.5\%) samples were positive on concentrated method which were negative on direct smear. More than $90 \%$ of the samples were found to be negative on both methods. Among 841 negative specimens on direct microscopy, $11(1.3 \%)$ and $3(0.4 \%)$ were found to be positive on concentrated methods. More than $66 \%$ of scanty, $52.6 \%$ of $1+$ and $37.1 \%$ of $2+$ AFB on direct microscopy were converted into $1+, 2+$ and $3+$ respectively on concentrated smear microscopy. No difference was found in case of high positive (3+) on both methods (Table 1 ).

Among the 915 specimens, 105 (11.5\%) were found to be positive on culture. Considering 105 culture positive cases, 74 (70.5\%) and $87(82.9 \%)$ of them were AFB positive in direct and concentrated smear, respectively (Table 2). About $17.1 \%$ culture positive cases were found to be negative on both direct and concentrated AFB microscopy.

The sensitivity of direct and concentrated smear microscopy was different when using positive culture result as the gold standard. Applying the McNemar $\chi^{2}$ test, the difference between sensitivities ( $71 \%$ versus $83 \%$; $p=0.002)$ obtained by the two methods was found to be significant. These results showed that concentrated technique increases the sensitivity of microscopy up to $12 \%$ when performed with the same specimens. Among the 810 patients with negative cultures, the specificity (100\%) was similar for both techniques.

\section{Discussion}

This study showed that the use of the concentrated method for preparing smears for AFB microscopy increases sensitivity without a loss of specificity in identifying positive TB cases, compared to the direct method.

The specimen that was positive with the direct method but negative with the concentrated method was also found to be positive by culture. It was an uncommon phenomenon and theoretically it is difficult to explain such cases. It might have occurred due to inappropriate sample concentration and smear preparation, smear preparation from a negative sample accidentally, faulty staining process, or inappropriate microscopic observation.
Eight specimens were found to be positive in both direct and concentrated smear microscopy but negative in culture. From the clinical data, it was observed that these patients were taking anti-TB drugs while collecting the specimens. During treatment, only the dead/killed bacteria remain in the respiratory specimen (sputum) which were detected by microscopy but were unable to grow in L-J media.

A study conducted by Barez, et al. [26] showed that the sensitivity was almost similar in both methods as described $81.6 \%$ for direct method and $82.7 \%$ for the concentrated method. In another study, Cattamanchi et al. [27] failed to find a difference in sensitivity between direct and concentrated sputum smear microscopy, the calculated sensitivity of direct and concentrated smear microscopy was not significantly different (51\% vs. $52 \%)$. In a similar study conducted by Peterson et al. [28] in two different laboratory settings (a tertiary-care laboratory and several local outpatients clinics) found that in a tertiary-care hospital the direct smear was significantly less sensitive than the concentrated smear $(28 \%$ and $51 \%$, respectively) and in the samples from outpatients of the Pacific islands the direct smear was less sensitive than that made from the concentrated specimen (82 versus $93 \%$, respectively).

Despite some evidence that the concentrated method can be superior to the direct method, it is not being performed in peripheral TB laboratories in low-income countries, because of the following concerns: feasibility of centrifugation in settings with irregular power supply; limited human and financial resources; inadequate training capacity; lack of proper biosafety arrangements; and potential biohazard posed by centrifugation. In most resource poor countries like Bangladesh where TB is endemic, most of the microscopy centers are using direct smear microscopy with low sensitivity for the diagnosis of TB. However, in the light of our study findings, it may be recommend that concentrated smear microscopy can be used in place of conventional direct microscopy where appropriate facility is available, to achieve better diagnostic accuracy and ensure greater success of the TB control programmes. It may not be recommended as an ideal alternative to existing conventional microscopy considering the cost implications and other resource

Table 2 Comparison of both microscopy methods with the gold standard culture method

\begin{tabular}{lcccccc}
\hline AFB microscopy & & Gold standard + & Gold standard - & Total & Sensitivity \% (95\% Cl) & Specificity \% (95\% Cl) \\
\hline Direct & + & 74 & 0 & 74 & $70.5(60.8-79.0)$ & $100(99.5-100)$ \\
& - & 31 & 810 & 841 & & $100(99.5-100)$ \\
Concentration & + & 87 & 0 & 87 & $82.9(73.2-88.7)$ & \\
& - & 18 & 810 & 828 & & \\
Total & 105 & 810 & 915 & & \\
\hline
\end{tabular}


constraints involved with its implementation in the wide network of Bangladesh NTP, and other high TB burden countries. As alternative approaches like recently developed LED-FM microscopy and low cost sedimentation technique have shown to increase the sensitivity of microscopy without the involvement of much of resource, these can be considered to be incorporated in the national TB control activity framework rather than concentrated smear technique. However, for the district level laboratories where minimum biosafety arrangements can be made by putting in low cost biosafety cabinets and other constraints can be overcome, this concentrated smearing technique can be practiced to ensure better case detection of TB. There is still scope of further research to compare the outputs of concentrated smear technique and LED-FM in settings where appropriate resources are available. Research may also be conducted to find out the benefit of combining concentrated sample and fluorescent staining technique. As both these methods are already documented to increase the sensitivity of smear microscopy, their combination may have incremental effect and have the potential to increase the sensitivity of microscopy significantly.

\section{Conclusion}

The study showed that concentrated AFB microscopy is more efficient to detect $M$. tuberculosis in respiratory specimens than direct AFB microscopy. Due to resource constraints it may not be suitable for implementing in the wide network of NTP microscopy centers thorough out the country but it may be considered for the district and other higher level TB diagnosis centers which have more resources available at their disposal to ensure greater success in TB case detection.

\section{Competing interests}

The authors declare that no competing interests exist.

\section{Authors' contributions}

Conceived and designed the experiments: SB FVL. Performed the experiments: MKMU MRC. Analyzed the data: SB MTR RK. Wrote the paper: SB MKMU SA MTR FvL. Performed the field work: SB MTR. Facilitated data collection in the prison: MTR. All authors read and approved the final manuscript.

\section{Acknowledgements}

We are thankful to the Directorate of Prisons who helped us in performing this study in this difficult setting. We also thank all the doctors and support staff of Dhaka Central Jail Hospital for their help and support. Our sincere gratitude goes to all the prison inmates who participated in this study to make it a success.

\section{Author details \\ ${ }^{1}$ International Centre for Diarrhoeal Disease Research, Bangladesh. 2Department of Global Health, Amsterdam Institute for Global Health and Development, Academic Medical Centre, University of Amsterdam, Amsterdam, The Netherlands.}

Received: 1 November 2012 Accepted: 23 July 2013 Published: 25 July 2013

\section{References}

1. World Health Organization: Global Tuberculosis Control: WHO Report. 2011. http://www.who.int/tb/publications/global_report/2011/gtbr11_full.pdf.

2. Robert K: WHO declares TB a global emergency. Health Horiz 1993, 19:251-252.

3. TB Control in Prisons - A Manual for Program Managers; WHO/CDS/TB/ 2000281. http://whqlibdoc.who.int/hq/2000/WHO_CDS_TB_2000.281.pdf.

4. Huebner RE, Good RC, Tokars Jl: Current practices in mycobacteriology: results of a survey of state public health laboratories. J Clin Microbiol 1993, 31(4):771-775.

5. Aber VR, Allen BW, Mitchison DA, Ayuma P, Edwards EA, Keyes AB: Quality control in tuberculosis bacteriology. 1. laboratory studies on isolated positive cultures and the efficiency of direct smear examination. Tubercle 1980, 61(3):123-133.

6. Albert $\mathrm{H}$ : Economic analysis of the diagnosis of smear-negative pulmonary tuberculosis in South Africa: incorporation of a new rapid test, FASTPlaqueTB, into the diagnostic algorithm. Int J Tuberc Lung Dis 2004, 8(2):240-247

7. Suarez PG, Floyd K, Portocarrero J, Alarcon E, Rapiti E, Ramos G, Bonilla C, Sabogal I, Aranda I, Dye C, et al: Feasibility and cost-effectiveness of standardised second-line drug treatment for chronic tuberculosis patients: a national cohort study in Peru. Lancet 2002, 359(9322):1980-1989.

8. Toman K, Frieden T: Toman's Tuberculosis: case detection, treatment, and monitoring: questions and answers. Occupational and Environmental Medicine 2005, 62(1):70.

9. Siddiqi $K$, Lambert $M-L$, Walley J: Clinical diagnosis of smear-negative pulmonary tuberculosis in low-income countries: the current evidence. Lancet Infect Dis 2003, 3(5):288.

10. Apers L, Mutsvangwa J, Magwenzi J, Chigara N, Butterworth A, Mason P, Van Der Stuyft P: A comparison of direct microscopy, the concentration method and the mycobacteria growth indicator tube for the examination of sputum for acid-fast bacilli. Int J Tubercul Lung Dis 2003, 7(4):376-381.

11. Getahun $H$, Harrington $M, O^{\prime}$ Brien $R$, Nunn P: Diagnosis of smear-negative pulmonary tuberculosis in people with HIV infection or AIDS in resourceconstrained settings: informing urgent policy changes. Lancet 2007, 369(9578):2042-2049.

12. Corbett EL, Watt CJ, Walker N, Maher D, Williams BG, Raviglione MC, Dye C: The growing burden of tuberculosis: global trends and interactions with the HIV epidemic. Arch Intern Med 2003, 163(9):1009-1021.

13. Steingart KR, Ramsay A, Pai M: Optimizing sputum smear microscopy for the diagnosis of pulmonary tuberculosis. Expert Rev Anti Infect Ther 2007, 5(3):327-331.

14. Kent PT, Kubica GP: Public health mycobacteriology: a guide for the level III laboratory. Atlanta, Ga: US Department of Health and Human Services, Public Health Service, Centers for Disease Control; 1985.

15. Heifets LB, Good RC: Current laboratory methods for the diagnosis of tuberculosis. Washington, DC: Tuberculosis: pathogenesis, protection, and control American Society for Microbiology; 1994:85-110.

16. Kent PT, Kubica GP: Public Health Mycobacteriology: A Guide for the Level III Laboratory. Atlanta, Ga: US Department of Health and Human Services, Centers for Disease Control; 1985:159-184.

17. Diseases IUATaL: Tuberculosis guide for low income countries. Paris, France: International Union Against Tuberculosis and Lung Diseases; 1996.

18. Angeby KA, Alvarado-Galvez C, Pineda-Garcia L, Hoffner SE: Improved sputum microscopy for a more sensitive diagnosis of pulmonary tuberculosis. Int J Tuberc Lung Dis 2000, 4(7):684-687.

19. Cattamanchi A, Davis J, Pai M, Huang L, Hopewell P, Steingart K: Does bleach processing increase the accuracy of sputum smear microscopy for diagnosing pulmonary tuberculosis? J Clin Microbiol 2010, 48(7):2433-2439.

20. Armstrong D: LeD-based fluoroscopy and the ParaLens system: illuminating the future of TB diagnostics. Tuberculosis 2009, 7:17-18.

21. Stop TB Partnership and World Health Organization: New laboratory diagnostic tools for tuberculosis control Retooling task force. Geneva, Switzerland: WHO; 2008. http://www.finddiagnostics.org/export/sites/default/resource-centre/ reports_brochures/docs/New_Lab_Diagnostic_Tools_for_TB.pdf.

22. Uy R, Yu C, Juco M, Adlawan C, Ruiz G, Velmonte M, Zaldivar C: Clorox concentration technique for the demonstration of acid fast bacilli in the sputum. J Microbiol 1988, 17(1):13-18.

23. Bass J Jr, Farer L, Hopewell P, Jacobs R, Snider D Jr: Diagnostic standards and classification of tuberculosis. Am J Respiratory Crit Care Med 1990, 142(3):725-735. 
24. Miorner H, Ganlov G, Yohannes Z, Adane Y: Improved sensitivity of direct microscopy for acid-fast bacilli: sedimentation as an alternative to centrifugation for concentration of tubercle bacilli. J Clin Microbiol 1996, 34(12):3206-3207.

25. Banu S, Hossain S, Uddin MKM, Rahman MT, Khatun R, Zaman K, Quaiyum M, van Leth F: Comparison of macroscopic and microscopic assessment of specimens collected for the diagnosis of tuberculosis. Open Infect Dis J 2012, 6:1-4.

26. Marie Yvette C, Barez MD, Myrna T, Mendoza MD, Regina S, Celada RMT, Heidi $R$, Santos RMT: Accuracy of AFB in relation to TB culture in detection of pulmonary tuberculosis. Phil J Microbiol Infect Dis 1995, 24(2):33-36.

27. Cattamanchi A, Dowdy DW, Davis JL, Worodria W, Yoo S, Joloba M, Matovu J, Hopewell PC, Huang L: Sensitivity of direct versus concentrated sputum smear microscopy in HIV-infected patients suspected of having pulmonary tuberculosis. BMC Infect Dis 2009, 9:53.

28. Peterson EM, Nakasone A, Platon-DeLeon JM, Jang Y, de La Maza LM, Desmond E: Comparison of direct and concentrated acid-fast smears to identify specimens culture positive for Mycobacterium spp. $J$ Clin Microbiol 1999, 37(11):3564-3568.

doi:10.1186/1756-0500-6-291

Cite this article as: Uddin et al: Comparison of direct versus concentrated smear microscopy in detection of pulmonary tuberculosis. BMC Research Notes 2013 6:291.

\section{Submit your next manuscript to BioMed Central and take full advantage of:}

- Convenient online submission

- Thorough peer review

- No space constraints or color figure charges

- Immediate publication on acceptance

- Inclusion in PubMed, CAS, Scopus and Google Scholar

- Research which is freely available for redistribution 